\title{
Elastic nuclear scattering at intermediate and relativistic energies
}

\author{
R. Matzdorf ${ }^{1}$, B. Fricke ${ }^{1}$, and G. Soff ${ }^{2}$ \\ ${ }^{1}$ Institut für Theoretische Physik, Universität Kassel, Heinrich-Plett-Strasse 40, W-3500 Kassel, \\ Federal Republic of Germany \\ ${ }^{2}$ Gesellschaft für Schwerionenforschung (GSI), Planckstrasse 1, Postfach 110552, W-6100 Darmstadt, \\ Federal Republic of Germany
}

Received 23 May 1990; final version 14 September 1990

\begin{abstract}
The classical scattering cross section of two colliding nuclei at intermediate and relativistic energies is reevaluated. The influence of retardation and magnetic field effects is taken into account. Corrections due to electron screening as well as due to attractive nuclear forces are discussed. This paper represents an addendum to $[1]$.
\end{abstract}

PACS: $24.10 .-\mathrm{i} ; 25.70 . \mathrm{Cd} ; 34.40 .+\mathrm{n}$

\section{Introduction}

The main goal of the new accelerator SIS at GSI is to explore atomic and nuclear physics processes in elastic and inelastic heavy-ion collisions with projectile energies up to $2 \mathrm{GeV} / \mathrm{u}$. In order to design experimental areas and to start an interpretation of the dominant processes it is necessary to reevaluate the elastic ion atom scattering cross section at intermediate and relativistic beam energies.

In a previous paper [1] we have already investigated the influence of relativistic and magnetic field effects as well as retardation corrections on elastic trajectories of heavy ions. We solved the classical equation of motion for a charged particle in an external electromagnetic field. In convariant form it reads

$\frac{\mathrm{d} p_{\alpha}}{\mathrm{d} \tau}=\frac{q}{c} F^{\alpha \beta} U_{\beta}$

where $p^{\alpha}$ and $U_{\beta}$ is the 4-momentum and the 4-velocity, respectively. $q$ denotes the charge and $\tau$ the proper time of the considered particle. $F^{\alpha \beta}$ is the electromagnetic field strength tensor. A computer code was developed which yields the nuclear trajectory in the laboratory frame as well as the corresponding ion scattering cross section.

The motivation for this supplementary note is twofold. First, additional deviations from the classical Rutherford scattering cross section caused by electron screen- ing and the attractive nucleus-nucleus force can now be taken into account. As can be seen below both effects are non-negligible quantities. Second, we should indicate a few corrections of the presented results in [1]:a) The unit at the ordinate of Figs. 1 and 4 must be modified from barn $/ \mathrm{sr}$ to $\mathrm{fm}^{2} / \mathrm{sr}$. $b$ ) In our original computer code the magnetic field produced by the collision partner entered with a wrong sign which has been corrected meanwhile.

\section{Nuclear forces and electron screening}

To examine the influence of nucleus-nucleus forces on elastic ion trajectories we employ a simple optical potential of Wood-Saxon type [2]

$$
\begin{aligned}
U(r)= & V_{c}(r)-V\left(1+\exp \left\{\frac{r-R_{v}}{a_{v}}\right\}\right)^{-1} \\
& +i W\left(1+\exp \left\{\frac{r-R_{w}}{a_{w}}\right\}\right)^{-1}
\end{aligned}
$$

with the radii

$$
\begin{aligned}
& R_{v}=r_{0 v}\left(A_{1}^{\frac{1}{3}}+A_{2}^{\frac{1}{3}}\right), \\
& R_{w}=r_{0 w}\left(A_{1}^{\frac{1}{3}}+A_{2}^{\frac{1}{3}}\right), \\
& R_{c}=r_{0 c}\left(A_{1}^{\frac{1}{3}}+A_{2}^{\frac{1}{3}}\right) .
\end{aligned}
$$

$V_{c}(r)$ denotes the Coulomb potential of a homogeneously charged sphere with radius $R_{c}$

$$
\begin{aligned}
& V_{c}(r)=\frac{Z_{1} Z_{2} e^{2}}{r} \quad \text { for } r>R_{c}, \\
& V_{c}(r)=\frac{Z_{1} Z_{2} e^{2}}{R_{c}}\left(\frac{3}{2}-\frac{1}{2} \frac{r^{2}}{R_{c}^{2}}\right) \quad \text { for } r<R_{c} .
\end{aligned}
$$

The imaginary part of potential (2) is not used in the classical evaluation of the elastic scattering cross section, 


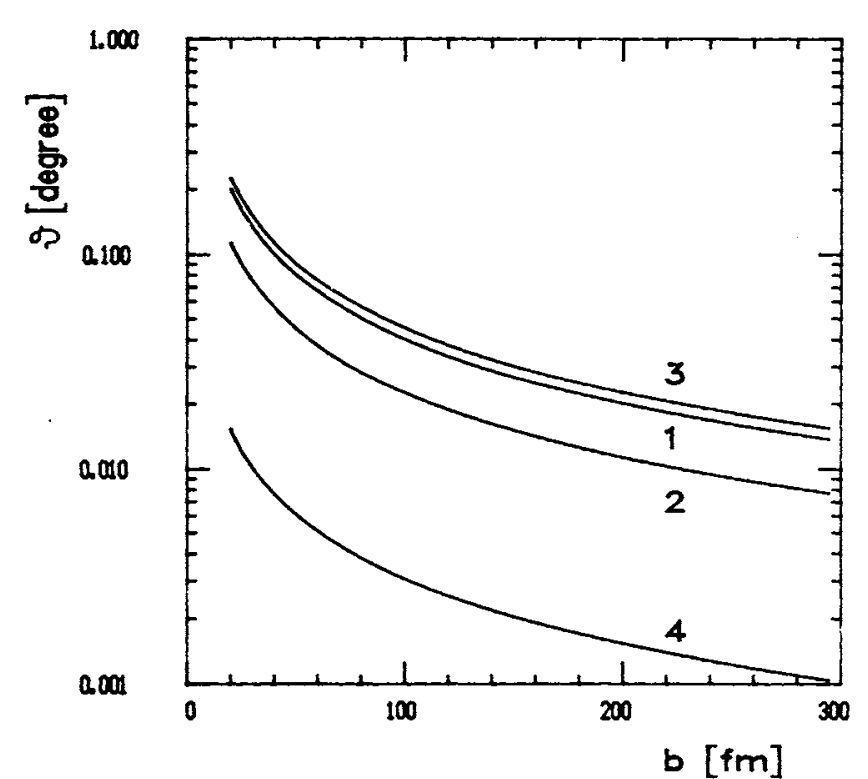

Fig. 1. Scattering angle (logarithmic scale) of the projectile in elastic collisions of heavy ions as function of the classical impact parameter $b$. The relativistic mass growth is taken into account. (1) ${ }^{208} \mathrm{~Pb}$ on ${ }^{238} \mathrm{U}$ at $1000 \mathrm{MeV} / \mathrm{u}$, (2) ${ }^{208} \mathrm{~Pb}$ on ${ }^{238} \mathrm{U}$ at $2000 \mathrm{MeV} / \mathrm{u}$, (3) ${ }^{9} \mathrm{Be}$ on ${ }^{238} \mathrm{U}$ at $1000 \mathrm{MeV} / \mathrm{u},(4){ }^{208} \mathrm{~Pb}$ on ${ }^{14} \mathrm{~N}$ at $1000 \mathrm{MeV} / \mathrm{u}$

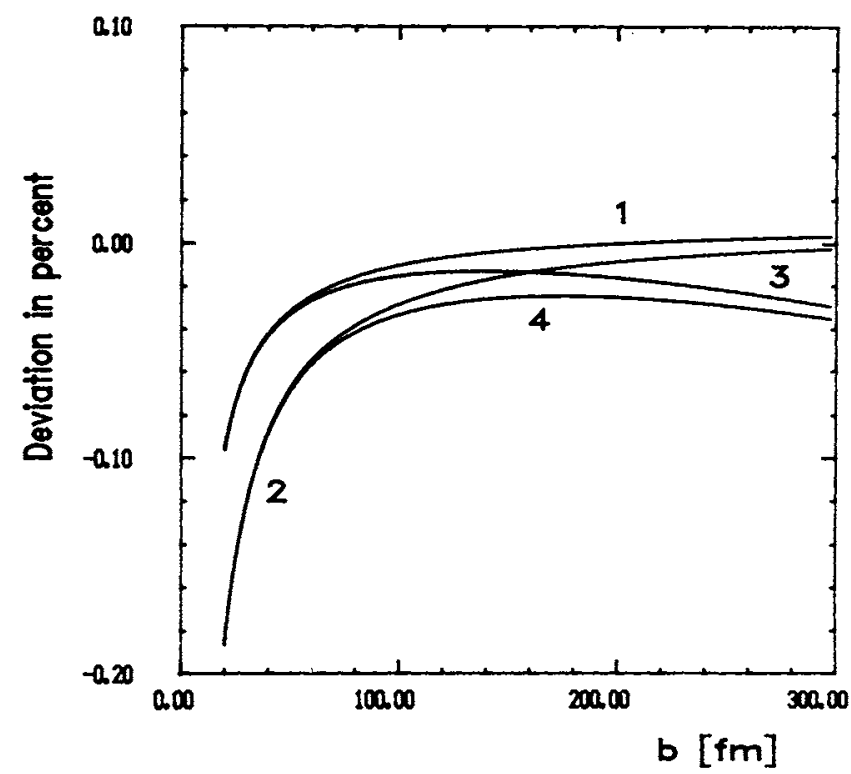

Fig. 2. The percentage deviation from the projectile scattering angle displayed for case (1) in Fig. 1. (1) Relativistic corrections. (2) Full relativistic calculation taking into account retardation and magnetic field effects. (3) The same as (1) but considering in addition the attractive nucleus-nucleus force and modifications caused by electron screening. (4) Sum of all additional effects

i.e. we fix $W=0$. In a quantum mechanical description of the scattering process a finite value of $W$ implies the possible absorption in the target. In the numerical solution of the equation of motion (1) we allow for accuracy tests of energy conservation during the collision. However, this is incompatible with a possible incorporation of energy loss as consequence of an imaginary potential.

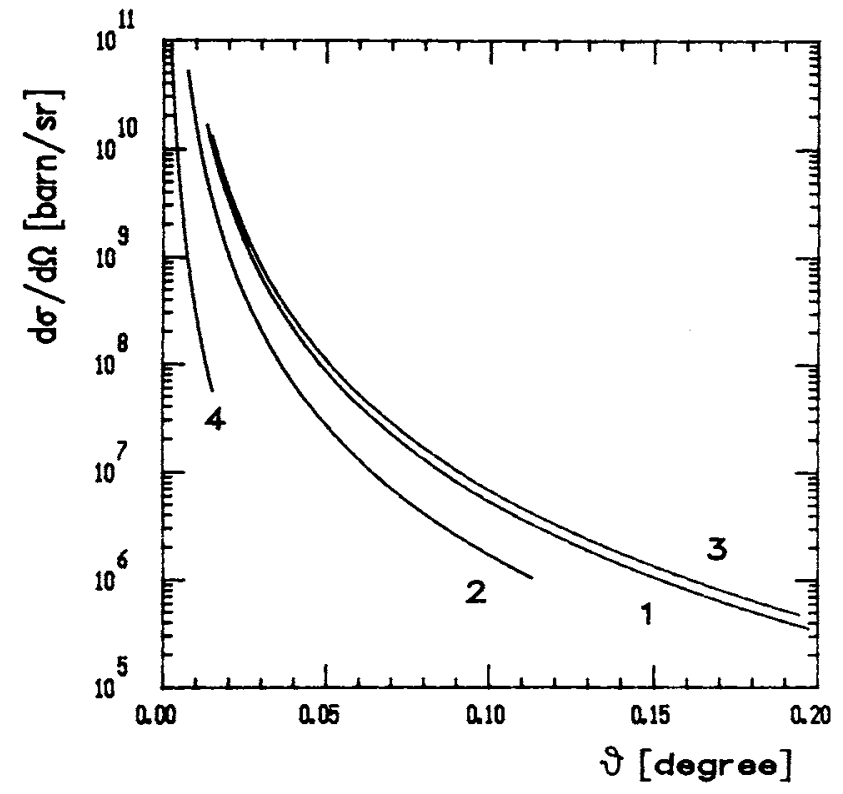

Fig. 3. Differential scattering cross section (logarithmic scale) in units of barn/sr for the systems (1) ${ }^{208} \mathrm{~Pb}$ on ${ }^{238} \mathrm{U}$ at $1000 \mathrm{MeV} / \mathrm{u}$, (2) ${ }^{208} \mathrm{~Pb}$ on ${ }^{238} \mathrm{U}$ at $2000 \mathrm{MeV} / \mathrm{u},(3){ }^{9} \mathrm{Be}$ on ${ }^{238} \mathrm{U}$ at $1000 \mathrm{MeV} / \mathrm{u}$, (4) ${ }^{208} \mathrm{~Pb}$ on ${ }^{14} \mathrm{~N}$ at $1000 \mathrm{MeV} / \mathrm{u}$

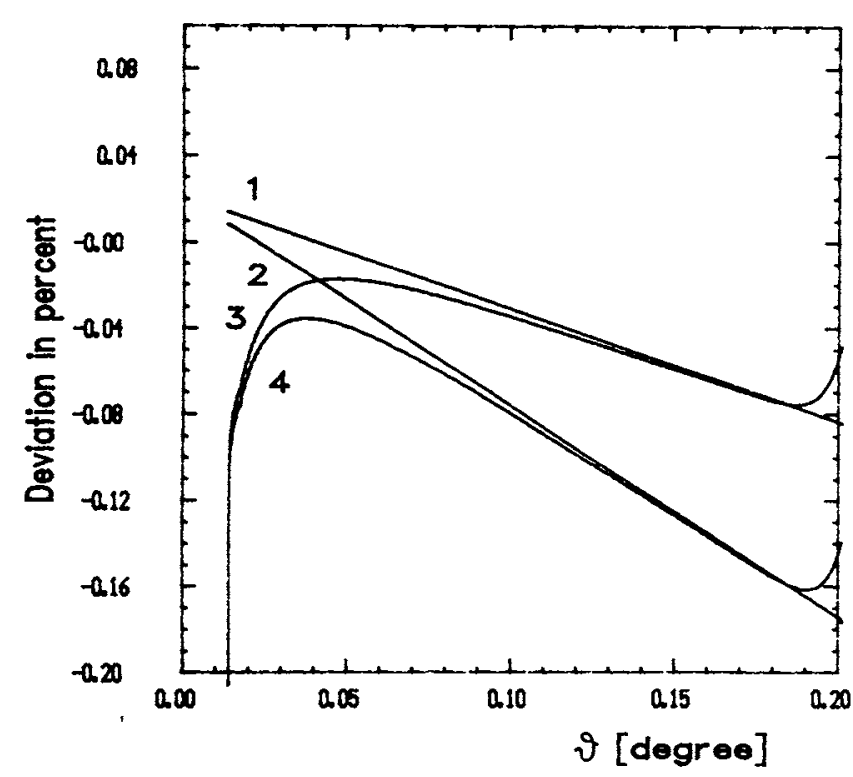

Fig. 4. Percentage deviation from the differential scattering cross section displayed for case (1) in Fig. 3. (1) Relativistic corrections. (2) Full relativistic calculation taking into account retardation and magnetic field effects. (3) The same as (1) but considering in addition the attractive nucleus-nucleus force and modifications caused by electron screening. (4) Sum of all additional effects

Typical parameters are

$r_{0 v}=r_{0 c}=1.2 \mathrm{fm}, \quad a_{v}=0.5 \mathrm{fm}, \quad V=100 \mathrm{MeV}$.

From (2) we obtain the force $\mathbf{F}(\mathbf{r})=-\nabla U(r)$ which enters the equation of motion (1) 


$$
\begin{aligned}
& F(r)=\frac{Z_{1} Z_{2} e^{2}}{r^{2}}-\frac{V \exp \left\{\frac{r-R_{v}}{a_{v}}\right\}}{a_{v}\left(1+\exp \left\{\frac{r-R_{v}}{a_{v}}\right\}\right)^{2}} \quad \text { for } r>R_{c}, \\
& F(r)=\frac{Z_{1} Z_{2} e^{2}}{R_{c}^{3}} r-\frac{V \exp \left\{\frac{r-R_{v}}{a_{v}}\right\}}{a_{v}\left(1+\exp \left\{\frac{r-R_{v}}{a_{v}}\right\}\right)^{2}} \quad \text { for } r<R_{c} .
\end{aligned}
$$

We restrict our considerations to impact parameters being large enough that nuclear forces either vanish or are relatively small which also implies that inelastic processes remain of minor importance.

To account for electron screening of the nuclear Coulomb potential we employ the Thomas-Fermi model [3] in an analytical parameterization of Molière [4]

$$
V_{c}(r)=\frac{Z_{1} Z_{2} e^{2}}{r} \phi(r)
$$

with the screening function

$\phi(r)=0.35 \mathrm{e}^{-0.3 r / a}+0.55 \mathrm{e}^{-1.2 r / a}+0.1 \mathrm{e}^{-6 r / a}$

and the screening length

$a=46847\left(Z_{1}^{\frac{1}{2}}+Z_{2}^{\frac{1}{2}}\right)^{-2 / 3} \mathrm{fm}$.

$a$ is of relatively large value which demonstrates that screening affects the elastic scattering already at large impact parameters. This yields the force due to the screened Coulomb potential

$$
\begin{aligned}
F_{c}(r)= & \frac{Z_{1} Z_{2} e^{2}}{r^{2}}\left[0.35 \mathrm{e}^{-0.3 r / a}+0.55 \mathrm{e}^{-1.2 r / a}+0.1 \mathrm{e}^{-6 r / a}\right] \\
& +\frac{Z_{1} Z_{2} e^{2}}{r a}\left[0.105 \mathrm{e}^{-0.3 r / a}+0.66 \mathrm{e}^{-1.2 r / a}\right. \\
& \left.+0.6 \mathrm{e}^{-6 r / a}\right]
\end{aligned}
$$

For the sake of completeness we mention that there are supplementary corrections to the elastic ion scattering cross section resulting e.g. from nuclear deformation, polarization of the nucleus and of the electronic cloud, vacuum polarization and additional radiative corrections. These effects which are supposed to be of minor importance are not taken into account in the present computations. Furthermore, since we stick to purely elastic collisions we neglected sizable contributions from inelastic processes such as nuclear Coulomb excitation, nucleon transfer, deep-inelastic nuclear reactions, ionization and pair creation. But again, the knowledge of elastic trajectories represents a necessary prerequisite for the evaluation of the cross section for these inelastic processes.

\section{Results}

To investigate the influence of the various contributions on the ion scattering cross section we have chosen as typical examples the following projectile - target combi- nations: a) heavy projectile - heavy target, ${ }^{208} \mathrm{~Pb}$ on ${ }^{238} \mathrm{U}$ at $1000 \mathrm{MeV} / \mathrm{u}$ and $2000 \mathrm{MeV} / \mathrm{u}, b$ ) light projectile - heavy target, ${ }^{9} \mathrm{Be}$ on ${ }^{238} \mathrm{U}$ at $1000 \mathrm{MeV} / \mathrm{u}, \mathrm{c}$ ) heavy projectile - light target, ${ }^{208} \mathrm{~Pb}$ on ${ }^{14} \mathrm{~N}$ at $1000 \mathrm{MeV} / \mathrm{u}$. The system ${ }^{132} \mathrm{Xe}-{ }^{238} \mathrm{U}$ considered in [1] falls into the category a).

Figure 1 displays the dependence of the projectile scattering angle $\vartheta$ as function of the classical impact parameter $b$. These curves include the effect of retardation the magnetic field effects as well as the effect of electron screening. But these additional influences are very small in absolute value. In order to indicate these effects we present Fig. 2 where one can deduce the percentage deviation from relativistic Rutherford scattering, in which the ordinary mass $m_{0}$ of the nucleus is replaced by its relativistic counterpart $\gamma m_{0}$ with the Lorentz contraction factor $\gamma$. For impact parameters $b$ smaller than the sum $R_{p}+R_{t}$ of the nuclear radii the nucleus-nucleus force is dominant. But in this region inelastic processes yield an overwhelming contribution to the scattering cross section and can no longer be neglected. In conclusion our results may not be applied for $b<R_{p}+R_{t}=b_{\mathrm{cr}}$. Already for impact parameters $b$ being a few fermis larger than $b_{\mathrm{cr}}$ the influence of the nucleus-nucleus force is completely negligible due to its exponential dependence on the radial coordinate $r$. The various curves 1 to 3 present the changes in percent or the scattering angle whereas curve 4 is the final sum of all contributions compared to the main effect. Figure 3 presents the differential cross sections $\mathrm{d} \sigma / \mathrm{d} \Omega$ of the elastic scattering as function of the scattering angle. Due to the smallness of the contributions Fig. 4 presents the influence of the various effects in percent of the main contribution.

There is a relatively big influence of electron screening on the nuclear trajectory even in the vicinity of the nuclear charge centres. The deviation of the scattering cross section resulting from the solution of the relativistic equation of motion compared with the approximative nonrelativistic calculation using $\gamma m_{0}$ for the effective mass turns out to be in the same order of magnitude as the retardation and magnetic field effects as can be deduced from Fig. 4. As expected its main contribution results for very small scattering angles which correspond to large impact parameters. In summary, however, we can state that retardation effects amount to less than $0.5 \%$ relative deviation for beam energies around $1 \mathrm{GeV} /$ u. More important is the contribution of the nucleusnucleus potential for impact parameters $b \sim b_{\text {cr }}$ as well as screening corrections for large impact parameters.

G.S. is grateful for fruitful discussions with H.J. Wollersheim.

\section{References}

1. Matzdorf, R., Mehler, G., Soff, G.: Z. Phys. D - Atoms, Molecules and Clusters 6, 5 (1987)

2. Bass, R.: Nuclear reactions with heavy ions. Berlin, Heidelberg, New York: Springer 1980

3. Thomas, L.H.: Proc. Cambridge Philos. Soc. 23, 542 (1927); Fermi, E.: Z. Phys. 48, 73 (1928)

4. Molière, G.: Z. Naturforsch. 2a, 133 (1947) 